\title{
Association between physical activity and metabolic syndrome in middle-aged Japanese: a cross-sectional study
}

\author{
Junghoon Kim¹,2, Kai Tanabe ${ }^{1}$, Noriko Yokoyama', Hirofumi Zempo ${ }^{1}$ and Shinya Kuno ${ }^{1,3^{*}}$
}

\begin{abstract}
Background: Although many studies have reported an association between self-reported physical activity and metabolic syndrome (MetS), there is limited information on the optimal level of physical activity required to prevent MetS. This study aimed to determine the association between objectively measured physical activity and MetS in middle-aged Japanese individuals. We also determined the optimal cutoff value for physical activity required to decrease the risk of developing MetS.

Methods: A total of 179 men and 304 women, aged between 30 and 64 years, participated in this study. Participants were divided into two groups using the Japanese criteria for MetS as those with MetS or pre-MetS, and those without MetS. Participants were considered to be physically active if they achieved a physical activity level of 23 metabolic equivalents (METs) h/week, measured using a triaxial accelerometer. The association between physical activity and MetS was analyzed using logistic regression with the following covariates: sex, age, sedentary time, low intensity activity, calorie intake, smoking, menopause and body mass index. We also evaluated the factors that determined the association between the prevalence of MetS and pre-MetS and the physical activity cutoff value using classification and regression tree (CART) analysis.

Results: The odds ratio for MetS and pre-MetS was 2.20 for physically inactive participants ( $<23 \mathrm{METs}$ h/week), compared with physically active participants ( $\geq 23$ METs h/week). The corresponding odds ratios for men and women were $2.27(P<0.01)$ and 1.95 (not significant), respectively. CART analyses revealed that moderate-vigorous physical activity of > 26.5 METs h/week was sufficient to decrease the prevalence of MetS and pre-MetS in middleaged Japanese men and women.
\end{abstract}

Conclusions: The results of this cross-sectional study indicate that the Exercise and Physical Activity Reference for Health Promotion 2006 is inversely associated with the prevalence of MetS in men. Our results also suggest that moderate physical activity of $>26.5$ METs h/week may decrease the risk of developing MetS and pre-MetS in middle-aged Japanese individuals.

\section{Background}

Metabolic syndrome (MetS) is defined as a cluster of risk factors that predispose individuals to atherosclerotic cardiovascular disease [1] and type 2 diabetes [2,3]. Individuals with MetS have higher cardiovascular disease mortality rates than do individuals without this syndrome $[4,5]$. Therefore, developing effective and

\footnotetext{
* Correspondence: kuno@taiiku.tsukuba.ac.jp

'Department of Sports Medicine, Graduate School of Comprehensive Human Sciences, University of Tsukuba, Tsukuba, Japan

Full list of author information is available at the end of the article
}

affordable strategies to combat MetS will be of great individual and societal importance.

Current epidemiological studies indicate that moderate-vigorous physical activity (MVPA) is associated with decreased risk of developing MetS, independent of aerobic fitness and obesity [6-11]. Other studies have also shown that daily physical activity prevents the onset of chronic diseases such as type 2 diabetes and MetS $[12,13]$. The Exercise and Physical Activity Reference for Health Promotion report 2006 (EPAR2006) compiles the current guidelines for health promotion and gives recommendations for physical activity and exercise for

\section{() Biomed Central}

(c) 2011 Kim et al; licensee BioMed Central Ltd. This is an Open Access article distributed under the terms of the Creative Commons Attribution License (http://creativecommons.org/licenses/by/2.0), which permits unrestricted use, distribution, and reproduction in any medium, provided the original work is properly cited. 
Japan $[14,15]$. These guidelines focus on daily physical activity exceeding 3 metabolic equivalents (METs), which includes both lifestyle physical activity and exercise, unlike the former exercise guidelines [15]. According to EPAR2006, every adult should accumulate 23 METs h/week of physical activity to prevent the onset of lifestyle-related diseases [14].

The guidelines for physical activity specified in EPAR2006 were established on the basis of a systematic review of studies performed mostly in Western countries, and included very few studies measuring physical activity in Japanese individuals. The Japanese population differs from the European and American populations in terms of genetic background and social environment. Furthermore, the guidelines were largely established using data obtained from a self-reported questionnaire survey. Such analyses are prone to recall error and overreporting, and are limited by their specific focus on MVPA [16,17]. Furthermore, it is difficult to accurately evaluate activities of daily life using a questionnaire [18]. Objective assessment of physical activity using accelerometers can overcome these limitations. This is supported by the results of a recent study that demonstrated a good construct validity for accelerometers compared with self-reported energy expenditure from physical activity [19].

Although many studies have reported an association between self-reported physical activity and MetS, there is limited information on the optimal level of physical activity required to prevent MetS and its associated cardiovascular abnormalities. Moreover, EPAR2006's physical activity parameters have not yet been correlated with MetS. Therefore, the objective of this study was to establish a physical activity reference relative to MetS in middle-aged Japanese men and women using an objective measurement of physical activity. We also determined the optimal cutoff value for physical activity required to decrease the risk for MetS.

\section{Methods}

\section{Participants}

The participants in this cross-sectional study were 521 healthy middle-aged Japanese volunteers without diabetes, cardiovascular disease, and musculoskeletal diseases recruited from local community newspapers in Tsukuba, Ibaraki. We excluded individuals who were missing data on physical activity $(\mathrm{n}=11)$, MetS components $(n=18)$ or dietary intake $(n=9)$. After excluding these individuals, a total of 179 men and 304 women were included in this analysis [age, 30-64 years; body mass index (BMI), $17.2-45.5 \mathrm{~kg} / \mathrm{m}^{2}$ ]. This study was approved by the Ethical Committee of the Institute of Health and Sport Sciences and the Institute of Clinical Medicine at the University of Tsukuba. Written informed consent was obtained from all participants. Participant characteristics $(n=483)$ are presented in Table 1.

\section{Anthropometric and body composition measurements}

Body weight was measured to the nearest $0.1 \mathrm{~kg}$ using a digital scale (TBF-551; Omron Healthcare Co., Ltd., Kyoto, Japan), and height to the nearest $0.1 \mathrm{~cm}$ using a wall-mounted stadiometer. BMI was calculated as body weight $/$ height ${ }^{2}\left(\mathrm{~kg} / \mathrm{m}^{2}\right)$. Waist circumference (WC) was measured three times to the nearest $0.1 \mathrm{~cm}$ at the midpoint between the lower costal margin and the iliac crest using a calibrated measuring tape. Body fat percentage ( $\%$ body fat) and muscle mass percentage ( $\%$ muscle mass) were calculated from the impedance value (HBF-352, Omron Healthcare Co., Ltd., Kyoto, Japan) [20].

\section{Measurement of physical activity and sedentary time}

A triaxial accelerometer (HJA-350IT, Active style Pro, Omron Healthcare Co., Ltd.) was used to measure physical activity and sedentary time [21]. To ensure a valid reflection of long-term day-to-day activities, the accelerometer was required to be worn by participants for 7 days while the subjects were continuing their regular daily activities [22]. The participants were instructed to wear the accelerometer on an elastic waist band on the right hip throughout the day from the time they woke up in the morning until they went to bed at night, except during showers and bathing. Participants who did not record 10 hours/day of activity for 7 days were excluded from further analyses [22]. The mean wear time was 11.8 \pm 1.6 hours/day. Accelerometer data were calculated as the monitoring time spent in each of three different intensity levels for 1 week, using software provided by the manufacturer (HMS-HJA-IC01J; Omron Healthcare Co., Ltd.) as follows: sedentary (1.1-1.4 METs), low (1.52.9 METs), and moderate-to-vigorous ( $\geq 3$ METs). To compare the impact of different levels of MVPA on the risk of MetS, we defined two categories of MVPA based on the physical activity reference recommended in the EPAR2006: < 23 METs h/week and $\geq 23$ METs h/week.

\section{Blood pressure and biochemical blood assay}

Blood pressure was measured twice using an electronic digital blood pressure monitor (SM-100; Omron Healthcare Co., Ltd.) with subjects in a seated position after a 10-min rest period. If the two readings differed by more than $5 \mathrm{mmHg}$, a third reading was taken and the lowest was used. Approximately $10 \mathrm{~mL}$ of blood was drawn from each subject after an overnight fast. Fresh samples were used for enzymatic analysis of triglyceride levels, while fasting plasma glucose levels were assayed using the glucose oxidase method. Serum high-density 
Table 1 Descriptive characteristics of study participants

\begin{tabular}{|c|c|c|c|}
\hline & Men $(n=179)$ & Women $(n=304)$ & $P$ value \\
\hline Age (years) & $46.6(0.6)$ & $48.6(0.5)$ & 0.015 \\
\hline Height (cm) & $170(0.5)$ & $157.7(0.3)$ & $<0.001$ \\
\hline Body weight (kg) & $73.9(0.9)$ & $63.7(0.6)$ & $<0.001$ \\
\hline BMI $\left(\mathrm{kg} / \mathrm{m}^{2}\right)$ & $25.6(0.3)$ & $25.6(0.2)$ & 0.896 \\
\hline$\%$ muscle mass (\%) & $30.4(0.2)$ & $23.7(0.3)$ & $<0.001$ \\
\hline$\%$ fat mass $(\%)$ & $25.1(0.4)$ & $34.0(0.4)$ & $<0.001$ \\
\hline \multicolumn{4}{|l|}{ Physical activity parameter* } \\
\hline Sedentary time (h/week) & $37.2(0.9)$ & $29.5(0.7)$ & $<0.001$ \\
\hline Low intensity activity (METs h/week) & $77.1(1.8)$ & $96.4(1.3)$ & $<0.001$ \\
\hline MVPA (METs h/week) & $24.5(0.8)$ & $25.7(0.6)$ & 0.252 \\
\hline \multicolumn{4}{|l|}{ Metabolic syndrome components } \\
\hline Abdominal obesity (\%) & 66.7 & 43.9 & $<0.001$ \\
\hline Hyperglycemia (\%) & 29.8 & 18.8 & 0.005 \\
\hline Hypertension (\%) & 54.2 & 47.7 & 0.168 \\
\hline Dyslipidemia (\%) & 33.5 & 14.5 & $<0.001$ \\
\hline Number of MetS components (n) & $1.9(0.1)$ & $1.3(0.1)$ & $<0.001$ \\
\hline Metabolic syndrome & & & $<0.001$ \\
\hline Pre-MetS (\%) & 18.4 & 17.8 & \\
\hline MetS $(\%)$ & 34.6 & 16.4 & \\
\hline MetS/pre-MetS (\%) & 53.1 & 34.2 & \\
\hline \multicolumn{4}{|l|}{ Daily nutritional intake $^{\dagger}$} \\
\hline Calorie intake (kcal/day) & $2126.1(24)$ & $1899.1(15.6)$ & $<0.001$ \\
\hline Carbohydrate intake (g/day) & $253.0(7)$ & $238.6(4.8)$ & 0.081 \\
\hline Protein intake (g/day) & $76.9(1.9)$ & $68.6(1.6)$ & 0.010 \\
\hline Fat intake (g/day) & $60.0(1.8)$ & $55.5(1.5)$ & 0.058 \\
\hline Smoker (\%) & 22.3 & 5.3 & $<0.001$ \\
\hline Postmenopausal (\%) & - & 52.6 & \\
\hline
\end{tabular}

Values are means (SE) or percentage. ${ }^{*}$ Adjusted for age, ${ }^{\dagger}$ adjusted for body weight. BMI, body mass index; METs, metabolic equivalents; MVPA, moderatevigorous physical activity; MetS, metabolic syndrome; SE, standard error. Abdominal obesity: waist circumference $\geq 85 \mathrm{~cm}$ for men and $\geq 90 \mathrm{~cm}$ for women; hyperglycemia: blood glucose $\geq 110 \mathrm{mg} / \mathrm{dL}$; hypertension: systolic blood pressure $\geq 130 \mathrm{mmHg}$ and/or diastolic blood pressure $\geq 85 \mathrm{mmHg}$, or treatment for previously diagnosed hypertension; dyslipidemia: triglyceride $\geq 150 \mathrm{mg} / \mathrm{dL}$ and/or high-density lipoprotein cholesterol level $<40 \mathrm{mg} / \mathrm{dL}$, or specific treatment for these lipid abnormalities.

lipoprotein cholesterol (HDL-C) was measured using heparin-manganese precipitation.

\section{Components of MetS}

The Japanese criteria for MetS were used to evaluate the prevalence of MetS and pre-MetS in this study [23,24]. Based on these criteria, to diagnose MetS, the subject must present with abdominal obesity, which is a mandatory criterion because it is a strong predictor of cardiovascular risk factors, in addition to two or more of the other components. For the diagnosis of pre-MetS, the subject must have abdominal obesity and one of the other components, including (1) WC $\geq 85 \mathrm{~cm}$ for men and $\geq 90 \mathrm{~cm}$ for women, which is considered an essential component; (2) dyslipidemia (triglyceride $\geq 150 \mathrm{mg}$ per $\mathrm{dL}$ and/or HDL-C level $<40 \mathrm{mg}$ per $\mathrm{dL}$, or specific treatment for these lipid abnormalities); (3) hypertension (systolic blood pressure $\geq 130 \mathrm{mmHg}$ and/or diastolic blood pressure $\geq 85 \mathrm{mmHg}$, or treatment of previously diagnosed hypertension); or (4) hyperglycemia (fasting plasma glucose $\geq 110 \mathrm{mg} / \mathrm{dL}$ ). In this study, we analyzed physical activity for MetS subjects and pre-MetS subjects in a single group (MetS/pre-MetS).

\section{Covariates}

A nutritionist assessed the daily nutritional intake of each subject, including carbohydrate, fat and protein, as well as the total calorie intake per day, by evaluating the patients' 3 -day weight and dietary records. The food data of the dietary records were converted to energy and nutrient data by the dietician and analyzed using Excel Eiyo-kun software (Ver. 4.0; Kenpaku Co., Ltd., Tokyo, Japan). Participants also completed a general health questionnaire, which recorded smoking and menopausal status.

\section{Statistical analysis}

Differences in physical characteristics between subjects with MetS/pre-MetS and subjects without MetS were 
tested using unpaired $t$-tests. Physical activity parameters and nutrient intake were analyzed in both groups using analysis of covariance (ANCOVA) with age or body weight as covariates, respectively.

Logistic regression analysis was used to predict MetS from the levels of physical activity based on the EPAR2006 reference ( $\geq 23$ METs h/week or $<23$ METs $\mathrm{h} /$ week); this was done separately for men and women. In all logistic regression models, age, level of low intensity activity, sedentary time, smoking, calorie intake and BMI were included as covariates. The total cohort model was also adjusted for sex. The women's model was adjusted for menopause. Multicollinearity between sedentary time, level of low intensity activity and MVPA was tested but was not significant.

Classification and regression tree analysis (CART) $[25,26]$ was used to establish the cutoff value for required physical activity and the best predictor for the prevalence of MetS. CART analysis is constructed by repeatedly splitting subsets of the data using all explanatory variables to create two subgroups. Explanatory variables with the best improvement are selected to split the data based on the improvement score, and are evaluated to identify the optimal cutoff point (continuous variable) or groups (nominal variables). We calculated $P$ values at each branch using $\chi^{2}$ tests. Values are expressed as means \pm standard error (SE) or as percentages. Values of $P<0.05$ were considered statistically significant. All data were analyzed using SPSS version 17 or R version 2.10.1 [27].

\section{Results}

This study included 483 Japanese individuals (men $37.1 \%$, women $62.9 \%$ ) aged between 30 and 64 years (Table 1). Overall, $53.1 \%$ of men and $34.2 \%$ of women had MetS or pre-MetS (Table 1). The prevalence of abdominal obesity, hyperglycemia, dyslipidemia, and MetS values was significantly higher in men than in women (Table 1). MVPA was not significantly different between men and women $(P=0.252)$.

Table 2 shows the characteristics of participants with MetS/pre-MetS and of participants without MetS, by sex. In both men and women, we found significant differences in body weight and BMI between individuals with MetS/pre-MetS and those without MetS (Table 2). As described in a previous study, we assessed possible covariates that were likely to influence the prevalence of MetS. Because there seems to be a strong age-dependence in the prevalence of MetS [28], we included age as a covariate to adjust the physical activity parameters. With the inclusion of age in the model, we found a significant difference in MVPA in both men and women (Table 2). Notably, age, sedentary time and low intensity physical activity were significantly associated with MetS/
pre-MetS, but only in women. In contrast, daily nutrition intake adjusted by body weight, including total energy intake, and total carbohydrate, fat and protein intake, was not different between MetS/pre-MetS and non-MetS in both men and women (Table 2).

Results of logistic regression analyses comparing the prevalence of MetS/pre-MetS across physical activity categories by sex are shown in Table 3. In all analyses, the physically active group that met the physical activity reference standard ( $\geq 23 \mathrm{METs}$ h/week) was used as the reference group. After adjusting for sex, age, low intensity activity, sedentary time, smoking, calorie intake and BMI, the risk of MetS/pre-MetS in the physically inactive group was significantly increased compared with that in the physically active group [Table 3 : odds ratio (OR), 2.20; 95\% CI, 1.7-3.83]. The risk for MetS among physically inactive men was significantly higher than that for physically active men after adjustment for age, sedentary time, low intensity activity, smoking, calorie intake and BMI (Table 3: OR, 2.27; 95\% CI, 1.22-6.29). In contrast, the risk for MetS in women was not significantly different between physically active and physically inactive women after adjustment for age, sedentary time, low intensity activity, smoking, calorie intake, BMI and menopausal status (Table 3: OR, 1.95; 95\% CI, 0.85-4.45).

As shown in Figure 1, eight factors (MVPA, sedentary time, low intensity activity, sex, age, smoking, calorie intake and menopausal status) were included in the CART analysis. The first table in Figure 1 shows that MVPA decreased the prevalence of MetS/pre-MetS in 199 of the 483 participants $(41.2 \%)$. The most important factor that decreased the prevalence of MetS/pre-MetS was an MVPA of $>26.5$ METs h/week $(P<0.001)$. The prevalence of MetS/pre-MetS was lower in 47 of the 185 participants with an MVPA of > 26.5 METs h/week (25.4\%) and in 152 of the 298 participants (51.0\%) with an MVPA of $\leq 26.5 \mathrm{METs}$ h/week. Age was used to subdivide the participants, regardless of whether activity of 26.5 METs $\mathrm{h} /$ week was achieved. In the subgroup with an MVPA of $>$ 26.5 METs h/week, the prevalence of MetS/pre-MetS was decreased in 10 of the 69 participants aged $\leq 44.5$ years (14.5\%) and in 37 of the 116 participants aged $>44.5$ years (31.9\%). In the $\leq 26.5 \mathrm{METs} \mathrm{h} /$ week group, age was also the most important factor for the split, with increased prevalence of MetS/pre-MetS in 79 of 189 (41.8\%) and in 73 of $109(67.0 \%)$ participants aged $\leq 51.5$ years and $>51.5$ years, respectively. Sedentary time, low intensity activity, smoking, calorie intake and menopausal status were not significant parameters in CART analysis.

\section{Discussion}

This study aimed to determine a physical activity reference value relative to MetS in middle-aged Japanese men and 
Table 2 Characteristics of participants with MetS/pre-MetS and those without MetS, by sex

\begin{tabular}{|c|c|c|c|c|c|c|}
\hline & \multicolumn{3}{|l|}{ Men } & \multicolumn{3}{|l|}{ Women } \\
\hline & $\begin{array}{l}\text { Without MetS } \\
(\mathrm{n}=85)\end{array}$ & MetS/pre-MetS ( $n=94)$ & $P$ value & $\begin{array}{l}\text { Without MetS } \\
(\mathrm{n}=200)\end{array}$ & MetS/pre-MetS $(n=104)$ & $P$ value \\
\hline Age (years) & $45.5(0.9)$ & $47.6(0.8)$ & 0.088 & $46.7(0.7)$ & $52.3(0.8)$ & $<0.001$ \\
\hline Height (cm) & $168.8(0.7)$ & $171(0.6)$ & 0.023 & $157.8(0.4)$ & $157.6(0.6)$ & 0.853 \\
\hline Body weight (kg) & $66.9(0.9)$ & $80.1(1.1)$ & $<0.001$ & $58.6(0.5)$ & $73.4(0.9)$ & $<0.001$ \\
\hline BMI $\left(\mathrm{kg} / \mathrm{m}^{2}\right)$ & $23.5(0.3)$ & $27.4(0.3)$ & $<0.001$ & $23.6(0.2)$ & $29.5(0.3)$ & $<0.001$ \\
\hline$\%$ muscle mass (\%) & $30.8(0.4)$ & $30.2(0.2)$ & 0.102 & $24.8(0.2)$ & $21.9(0.5)$ & $<0.001$ \\
\hline$\%$ fat mass $(\%)$ & $23.3(0.6)$ & $26.5(0.4)$ & $<0.001$ & $31.7(0.4)$ & $37.7(0.4)$ & $<0.001$ \\
\hline \multicolumn{7}{|l|}{ Physical activity parameters* } \\
\hline Sedentary time (h/week) & $36.5(1.4)$ & $38.2(1.3)$ & 0.367 & $27.6(0.8)$ & $32.9(1.1)$ & 0.001 \\
\hline Low intensity activity (METs h/week) & $75.5(2.8)$ & $77.9(2.6)$ & 0.538 & $99.5(1.6)$ & $90.9(2.2)$ & 0.002 \\
\hline MVPA (METs h/week) & $28.4(1.2)$ & $21.0(1.2)$ & $<0.001$ & $27.6(0.7)$ & $23(1.0)$ & 0.001 \\
\hline \multicolumn{7}{|l|}{ Metabolic syndrome components } \\
\hline Waist circumference $(\mathrm{cm})$ & $82.6(0.6)$ & $93.4(0.9)$ & $<0.001$ & $83.1(0.5)$ & $100.9(0.8)$ & $<0.001$ \\
\hline $\mathrm{SBP}(\mathrm{mmHg})$ & $122.7(1.4)$ & $136.7(1.3)$ & $<0.001$ & $118.9(0.9)$ & $136.4(1.9)$ & $<0.001$ \\
\hline $\mathrm{DBP}(\mathrm{mmHg})$ & $79.1(1.0)$ & $90.5(1.1)$ & $<0.001$ & $79.1(0.7)$ & $91.8(1.1)$ & $<0.001$ \\
\hline Fasting glucose (mg/dL) & $100.3(1.0)$ & $109.7(1.6)$ & $<0.001$ & $97.2(0.8)$ & $115.1(3.1)$ & $<0.001$ \\
\hline Triglyceride (mg/dL) & $93.2(5.7)$ & $173.0(9.8)$ & $<0.001$ & $82.6(3.1)$ & $139.4(8.3)$ & $<0.001$ \\
\hline $\mathrm{HDL}-\mathrm{C}(\mathrm{mg} / \mathrm{dL})$ & $61.7(1.2)$ & $53.2(1.5)$ & $<0.001$ & $67.3(1.0)$ & $59.1(1.3)$ & $<0.001$ \\
\hline Number of MetS components ( $n$ ) & $0.7(0.1)$ & $2.9(0.1)$ & $<0.001$ & $0.6(0.0)$ & $2.6(0.1)$ & $<0.001$ \\
\hline \multicolumn{7}{|l|}{ Daily nutrition intake $^{\dagger}$} \\
\hline Calorie intake (kcal/day) & $2122.2(32.1)$ & $2129.6(35.4)$ & 0.878 & $1897.8(20.6)$ & $1901.4(22.6)$ & 0.913 \\
\hline Carbohydrate intake (g/day) & $252.7(11.4)$ & $253.3(9.0)$ & 0.971 & $241(6.1)$ & $233.6(7.9)$ & 0.471 \\
\hline Protein intake (g/day) & $74.5(3.1)$ & $78.6(2.4)$ & 0.292 & $68.9(1.9)$ & $67.9(3)$ & 0.753 \\
\hline Fat intake (g/day) & $59.8(2.6)$ & $60.1(2.4)$ & 0.948 & $56.7(1.7)$ & $53.2(2.9)$ & 0.271 \\
\hline Smoker (\%) & 17.9 & 26.3 & 0.175 & 5.5 & 4.8 & 0.798 \\
\hline Postmenopausal (\%) & - & - & & 45.0 & 67.3 & $<0.001$ \\
\hline
\end{tabular}

Values are means (SE) or percentage. *Adjusted for age, ${ }^{\dagger}$ adjusted for body weight. MetS, metabolic syndrome; BMI, body mass index; METs, metabolic equivalent; MVPA, moderate-vigorous physical activity; SBP, systolic blood pressure; DBP, diastolic blood pressure; HDL-C, high density lipoprotein cholesterol; SE, standard error.

women, to establish an appropriate cutoff value for physical activity that, if met, will decrease the prevalence of MetS. The results suggest that the EPAR2006 physical activity reference was significantly associated with MetS in men but not in women after adjustment for covariates. Moreover, we found that the cutoff value of physical activity required to decrease the risk of MetS was 26.5 METs $\mathrm{h} /$ week in middle-aged Japanese individuals.

Table 3 Odds ratios for the prevalence of metabolic syndrome according to physical activity levels

\begin{tabular}{lcc}
\hline MetS/pre-MetS & Univariate YCrude OR (95\% Cl) & Multivariate Adjusted OR (95\% Cl) \\
\hline Total $(n=483)$ & 1 & 1 \\
$\quad \geq 23$ METs h/week & $2.20(1.52-3.19)$ & $2.20(1.27-3.83)^{*}$ \\
$\quad<23$ METs h/week & 1 & 1 \\
\hline Men $(n=179)$ & $2.27(1.24-4.15)$ & $2.27(1.22-6.29)^{\dagger}$ \\
$\quad \geq 23$ METs h/week & 1 & 1 \\
$\quad<23$ METs h/week & $1.97(1.22-3.20)$ & $1.95(0.85-4.45)^{\ddagger}$ \\
\hline Women $(n=304)$ & & \\
$\quad \geq 23$ METs h/week &
\end{tabular}

${ }^{*}$ Adjusted for sex, age, calorie intake, smoking, sedentary time, level of low intensity activity and body mass index (BMI); ${ }^{\dagger}$ adjusted for age, calorie intake, smoking, sedentary time, level of low intensity activity and BMl; ${ }^{\ddagger}$ adjusted for age, calorie intake, smoking, sedentary time, level of low intensity activity, BMI and menopause. OR, odds ratios; $\mathrm{Cl}$, confidence interval. 


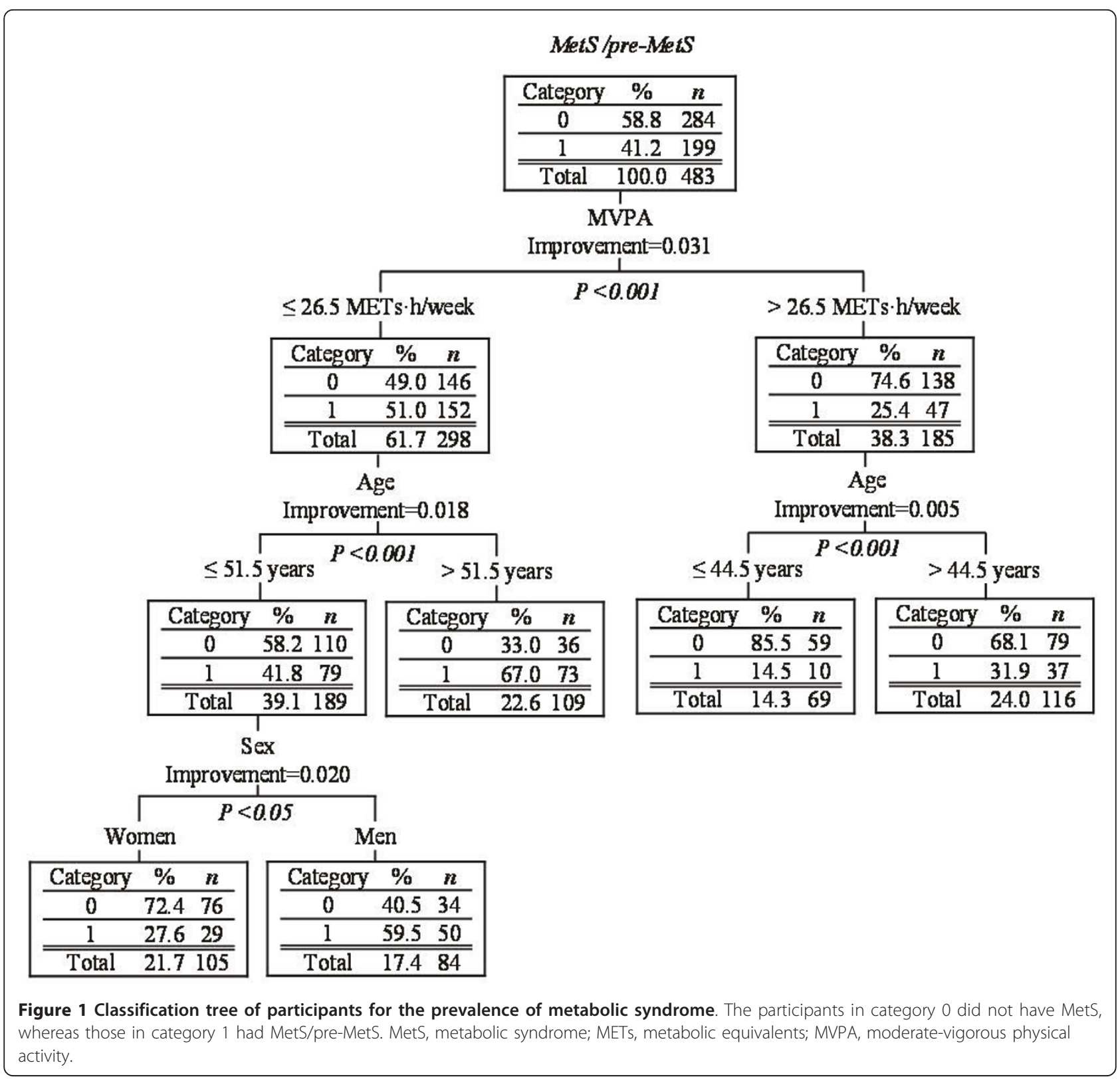

\section{Association between physical activity levels specified by EPAR2006 and MetS}

This present study is the first, to our knowledge, to examine the association between the EPAR2006-recommended physical activity levels and MetS. The analysis showed that physically active men ( $\geq 23$ METs h/week) were at less risk of MetS than their physically inactive counterparts (<23 METs h/week). In contrast, the association between MVPA and MetS in women was less clear. Our physical activity findings were similar to those reported by other similarly designed studies [6-8]. Contemporary analyses have shown that leisure-time physical activity is effective in preventing and treating
MetS [7,29], and most studies have shown that MetS is inversely associated with physical activity or physical fitness in men $[6,30,31]$. In contrast, few studies have observed inverse associations between physical activity and MetS in women [32].

Our study confirms the results of other studies showing an inverse association between physical activity and MetS, which applies particularly to men. Zhu et al. [33] reported a significant association between physical activity and MetS risk factors in men, but not in women, after considering covariates like age, race, income level and education. Moreover, the prevalence of MetS was $31 \%$ lower in physically active men than in their inactive 
counterparts, whereas the corresponding percentage among women was $17 \%$ [33].

Another study has noted differences between men and women in the association between physical activity or fitness and MetS [34]. Brien et al. [6] reported a significant association between physical activity and MetS in men, but not in women, after adjusting for age, smoking, alcohol consumption and income level. The authors attributed these sex differences to hormonal differences, varying patterns of fat distribution, differences in the definition of MetS, and differences in the types and intensities of physical activity $[8,34]$. Therefore, the results of the present study and those of other investigations may represent a true difference between sexes in terms of the association between physical activity and MetS.

\section{Cutoff value for the required amount of physical activity to prevent the onset of MetS}

This study also aimed to establish a cutoff value for the required amount of physical activity that would prevent MetS in middle-aged Japanese individuals. Although many studies have included cross-sectional analyses $[6,9,13,30,31]$, our study was the first, to our knowledge, to examine the optimal cutoff value of physical activity required to prevent the onset of MetS. CART analysis revealed that the optimal cutoff value of 26.5 METs h/ week represented the level of physical activity required to prevent MetS, and was slightly higher than the value reported by EPAR2006 (23 METs h/week) to prevent lifestyle-related disease $[14,15]$.

Furthermore, applying the EPAR2006 physical activity recommendations resulted in a MetS prevalence of $41.0 \%$ (data not shown), which was much higher than that determined in our study (25.4\%) when we applied the new cutoff value for physical activity (Figure 1). These results suggest that the EPAR2006 recommendations for physical activity should be increased to reduce the prevalence of MetS among middle-aged Japanese individuals. Our results do not negate the current EPAR2006 physical activity reference value, and the discrepancy between the physical cutoff values can be explained. First, the EPAR2006 physical activity reference was established using a systematic review that primarily summarized the evidence on the effects of physical activity on lifestyle-related disease, rather than focusing on MetS. Second, this systematic review included studies performed in Western countries, and relatively few studies in Japanese individuals were included. Third, the techniques used to measure physical activity may have differed. However, the present study established an association between objectively measured physical activity and MetS in Japanese individuals. For these reasons, the findings of this study might have an important influence on effective promoting physical activity focused on preventing MetS among middle-aged Japanese individuals.

\section{Strengths and limitations}

The primary strength of our study was that we used a triaxial accelerometer to objectively measure physical activity and sedentary times. However, there are several limitations to be discussed. First, it is not possible to infer causality or specify the direction of the observed effects because of the cross-sectional study design. Second, prevalence of MetS/pre-MetS was higher in this study subjects (men: $53.1 \%$, women: $34.2 \%$ ) compared with general Japanese adults in the National Health and Nutrition Examination Survey in 2008 (men: 45.3\%, women: $18.6 \%)$. Therefore, the results in this study may not generalize to the Japanese population. Although we controlled for several covariates, there may be other unexplored residual confounding variables such as genetic variation and sociocultural factors that may partly explain our findings. Nevertheless, we considered factors directly related to MetS prevalence, including sedentary time, low intensity physical activity, calorie intake, smoking, menopause status and BMI [35-38], in this study. The study sample size may also be a limitation, but it was sufficiently large to allow us to perform CART analyses to determine the optimal cutoff value for physical activity necessary to prevent MetS. Despite these limitations, our findings help us to understand the association between physical activity and MetS in middle-aged Japanese individuals.

\section{Conclusions}

In conclusion, the results of this study indicate that the EPAR2006 physical activity reference value was associated with MetS/pre-MetS in men. Moreover, we found that moderate physical activity of > 26.5 METs h/week may decrease the risk of MetS in middle-aged Japanese individuals. The validity of the cutoff value for physical activity and the efficacy of physical activity programs that focus on the factors described here to prevent and treat MetS will need to be confirmed in longitudinal and interventional studies.

\section{Acknowledgements}

We wish to thank the study participants and the practice teams for their collaboration and help with recruitment. We also thank Dr. Oshima for advice on physical activity measurement and Dr. Tsubaki for assistance with statistical analysis. This work was supported in part by the Japan Society for the Promotion of Science (JSPS, No. 23 1254).

\section{Author details}

'Department of Sports Medicine, Graduate School of Comprehensive Human Sciences, University of Tsukuba, Tsukuba, Japan. ${ }^{2}$ Japan Society for the Promotion of Science, Tokyo, Japan. ${ }^{3}$ Tsukuba Wellness Research. Co., Ltd., Tsukuba, Japan. 


\section{Authors' contributions}

JK helped design the study, was responsible for data collection and analysis, and helped to write the manuscript. KT and NY transformed the accelerometer data and provided advice on statistical analyses. $\mathrm{HZ}$ helped to measure physical activity. SK was jointly responsible for the concept and design of the study. All authors read and approved the final version of the manuscript for publication.

\section{Competing interests}

The authors declare that they have no competing interests.

Received: 6 April 2011 Accepted: 5 August 2011

Published: 5 August 2011

\section{References}

1. Reaven GM: Role of insulin resistance in human disease. Diabetes 1998, 37:1595-1607.

2. Laaksonen DE, Lakka HM, Niskanen LK, Kaplan GA, Salonen JT, Lakka TA: Metabolic syndrome and development of diabetes mellitus: application and validation of recently suggested definitions of the metabolic syndrome in a prospective cohort study. Am J Epidemiol 2002, 156:1070-1077.

3. Lorenzo C, Okoloise M, Williams K, Stern MP: The metabolic syndrome as predictor of type 2 diabetes: the San Antonio heart study. Diabetes Care 2003, 26:3153-3159.

4. Lakka HM, Laaksonen DE, Lakka TA, Niskanen LK, Kumpusalo E, Tuomilehto J, Salonen JT: The metabolic syndrome and total and cardiovascular disease mortality in middle-aged men. JAMA 2002, 288:2709-2716

5. Hu G, Qiao Q, Tuomilehto J, Balkau B, Borch-Johnsen K, Pyorala K Prevalence of the metabolic syndrome and its relation to all-cause and cardiovascular mortality in nondiabetic European men and women. Arch Intern Med 2004, 164:1066-1076.

6. Brien SE, Katzmarzyk PT: Physical activity and the metabolic syndrome in Canada. Appl Physiol Nutr Metab 2006, 31:40-47.

7. Lakka TA, Laaksonen DE: Physical activity in prevention and treatment of the metabolic syndrome. Appl Physiol Nutr Metab 2007, 32:76-88.

8. Yang X, Telama R, Hirvensalo M, Mattsson N, Viikari JS, Raitakari OT: The longitudinal effects of physical activity history on metabolic syndrome. Med Sci Sports Exerc 2008, 40:1424-1431.

9. Rennie KL, McCarthy N, Yazdgerdi S, Marmot M, Brunner E: Association of the metabolic syndrome with both vigorous and moderate physical activity. Int J Epidemiol 2003, 32:600-606.

10. Franks PW, Ekelund U, Brage S, Wong MY, Wareham NJ: Does the association of habitual physical activity with the metabolic syndrome differ by level of cardiorespiratory fitness? Diabetes Care 2004 27:1187-1193.

11. Ekelund U, Brage S, Franks PW, Hennings S, Emms S, Wareham NJ: Physical activity energy expenditure predicts progression toward the metabolic syndrome independently of aerobic fitness in middle-aged healthy Caucasians: the Medical Research Council Ely Study. Diabetes Care 2005, , 28: $1195-1200$

12. Laaksonen DE, Lindström J, Lakka TA, Eriksson JG, Niskanen L, Wikström K Aunola S, Keinänen-Kiukaanniemi S, Laakso M, Valle TT, llanne-Parikka P, Louheranta A, Hämäläinen H, Rastas M, Salminen V, Cepaitis Z, Hakumäki M, Kaikkonen H, Härkönen P, Sundvall J, Tuomilehto J, Uusitupa M, Finnish diabetes prevention study: Physical activity in the prevention of type 2 diabetes: the Finnish diabetes prevention study. Diabetes 2005, 54:158-165.

13. Méndez-Hernández $P$, Flores $Y$, Siani $C$, Lamure M, Dosamantes-Carrasco LD, Halley-Castillo E, Huitrón G, Talavera JO, Gallegos-Carrillo K, Salmerón J: Physical activity and risk of metabolic syndrome in an urban Mexican cohort. BMC Public Health 2009, 9:276.

14. General Affairs Division, Health Service Bureau, Ministry of Health, Labour and Welfare of Japan: Exercise and physical activity reference quantity for health promotion 2006 (EPAR2006)-Physical activity, Exercise, and Physical Fitness.[http://www.nih.go.jp/eiken/programs/pdf/epar2006.pdf].

15. Ishikawa-Takata K, Tabata I: Exercise and Physical Activity Reference for Health Promotion 2006 (EPAR2006). J Epidemiol 2007, 17:177.

16. Sallis J, Saelens B: Assessment of physical activity by self-report: status, limitations, and future directions. Res Q Exerc Sport 2000, 71:1-14.
17. Shephard RJ: Limits to the measurement of habitual physical activity by questionnaires. Br J Sports Med 2003, 37:197-206.

18. Donahoo W, Levine J, Melanson E: Variability in energy expenditure and its components. Curr Opin Clin Nutr 2004, 7:599-605.

19. Harris T, Owen C, Victor C, Adams R, Ekelund U, Cook D: A comparison of questionnaire, accelerometer, and pedometer: measures in older people. Med Sci Sport Exer 2009, 41:1392-1402.

20. Oshima Y, Shiga T, Namba H, Kuno S: Estimation of whole-body skeletal muscle mass by bioelectrical impedance analysis in the standing position. Obes Res Clin Pract 2010, 4:1.

21. Oshima Y, Kawaguchi K, Tanaka S, Ohkawara K, Hikihara Y, IshikawaTakata K, Tabata I: Classifying household and locomotive activities using a triaxial accelerometer. Gait Posture 2010, 31:370-374.

22. Trost SG, Pate RR, Freedson PS, Sallis JF, Taylor WC: Using objective physical activity measures with youth: how many days of monitoring are needed? Med Sci Sports Exerc 2000, 32:426-431.

23. The Examination Committee of Criteria for 'Metabolic Syndrome' in Japan: Criteria for 'metabolic syndrome' in Japan. J Jpn Soc Int Med 2005, 94:188-203, in Japanese.

24. Fujita T: The metabolic syndrome in Japan. Nat Clin Pract Cardiovasc 2008, 5:15-18.

25. Breiman LFJ, Olshen RA, Stone CJ: Classification and Regression Trees. Belmont, CA: Chapman \& Hall/CRC; 1984.

26. Nakata Y, Okura T, Matsuo T, Tanaka K: Factors alleviating metabolic syndrome via diet-induced weight loss with or without exercise in overweight Japanese women. Prev Med 2009, 48:351-356.

27. R Development Core Team: R: A Language and Environment for Statistical Computing. R Foundation for Statistical Computing, Vienna Austria. 2009 [http://www.R-project.org], ISBN 3-900051-07-0.

28. Cornier MA, Dabelea D, Hernandez TL, Lindstrom RC, Steig AJ, Stob NR, Van Pelt RE, Wang H, Eckel RH: The metabolic syndrome. Endocr Rev 2008, 29:777-822.

29. Carroll S, Dudfield M: What is the relationship between exercise and metabolic abnormalities? A review of the metabolic syndrome. Sports Med 2004, 34:371-418.

30. Byberg L, Zethelius B, McKeigue PM, Lithell HO: Changes in physical activity are associated with changes in metabolic cardiovascular risk factors. Diabetologia 2001, 44:2134-2139.

31. Carroll S, Cooke CB, Butterly RJ: Metabolic clustering, physical activity and fitness in nonsmoking, middle-aged men. Med Sci Sports Exerc 2000, 32:2079-2086.

32. Irwin ML, Ainsworth BE, Mayer-Davis EJ, Addy CL, Pate RR, Durstine JL: Physical activity and the metabolic syndrome in a tri-ethnic sample of women. Obes Res 2002, 10:1030-1037.

33. Zhu S, St-Onge MP, Heshka S, Heymsfield SB: Lifestyle behaviors associated with lower risk of having the metabolic syndrome. Metabolism 2004, 53:1503-1511.

34. Boule NG, Bouchard C, Tremblay A: Physical fitness and the metabolic syndrome in adults from the Quebec Family Study. Can J Appl Physiol 2005, 30:140-156.

35. Riccardi G, Giacco R, Rivellese AA: Dietary fat, insulin sensitivity and the metabolic syndrome. Clin Nutr 2004, 23:447-456.

36. Ishizaka N, Ishizaka Y, Toda E, Nagai R, Yamakado M: Association between cigarette smoking, white blood cell count, and metabolic syndrome as defined by the Japanese criteria. Intern Med 2007, 46:1167-1170.

37. McNeill AM, Rosamond WD, Girman CJ, Golden SH, Schmidt MI, East HE, Ballantyne $\mathrm{CM}$, Heiss $\mathrm{G}$ : The metabolic syndrome and 11-year risk of incident cardiovascular disease in the atherosclerosis risk in communities study. Diabetes Care 2005, 28:385-390.

38. Eshtiaghi R, Esteghamati A, Nakhjavani M: Menopause is an independent predictor of metabolic syndrome in Iranian women. Maturitas 2009, 65:262-266.

\section{Pre-publication history}

The pre-publication history for this paper can be accessed here: http://www.biomedcentral.com/1471-2458/11/624/prepub

doi:10.1186/1471-2458-11-624

Cite this article as: Kim et al:: Association between physical activity and metabolic syndrome in middle-aged Japanese: a cross-sectional study. BMC Public Health 2011 11:624 